\title{
Neutrino spin light efficiency in Gamma-Ray Bursts
}

\section{Alexander Grigoriev}

Skobeltsyn Institute of Nuclear Physics, Moscow State University, 119992 Moscow, Russia Department of Theoretical Physics, Moscow Institute of Physics and Technology, 141700

Dolgoprudny, Russia

\section{Alexey Lokhov}

Institute for Nuclear Research, Russian Academy of Sciences, 117312 Moscow, Russia

\section{Alexander Studenikin*}

Department of Theoretical Physics, Moscow State University, 119992 Moscow, Russia

Dzhelepov Laboratory of Nuclear Problems, Joint Institute for Nuclear Research, 141980

Dubna, Russia

E-mail: studenikesrd.sinp.msu.ru

\section{Alexei Ternov}

Department of Theoretical Physics, Moscow Institute of Physics and Technology, 141700

Dolgoprudny, Russia

The spin light of neutrino $(S L v)$ is a new recently introduced mechanism of electromagnetic radiation emitted by a massive neutrino (with a nonzero magnetic moment) moving in external media. Although this effect is very weak due to smallness of the neutrino magnetic moment, it can be of interest for astrophysical environments involving compact relativistic objects because its efficiency is higher, the higher the neutrino energy and background matter density. In this note we summarize conditions for best $S L v$ efficiency in astrophysical settings and conclude that the most suitable astrophysical site for manifestation of this phenomenon is represented by short Gamma-Ray Bursts (sGRBs) where generation of ultra-high energy neutrinos is anticipated and the matter density can be of the order of the nuclear density. We also give an estimation of the $S L v$ efficiency in the sGRB environment.

The 39th International Conference on High Energy Physics (ICHEP 2018)

4-11 July, 2018

Seoul, Korea

\footnotetext{
* Speaker.
} 
The spin light of neutrino [四] originates from the neutrino-photon magnetic moment coupling and from splitting of the neutrino energy over the helicity $s= \pm 1$, induced by external environment

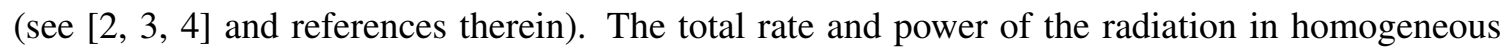
neutron matter (far from the threshold, see below),

$$
\Gamma=2 \mu^{2} G_{F}^{2} n^{2} p, \quad I={ }^{2} / 3 \mu^{2} G_{F}^{2} n^{2} p^{2},
$$

exhibit strong dependance on the neutrino energy and matter density $n$ ( $p$ and $\mu$, are the neutrino momentum and magnetic moment). The $S L v$ radiation has also a non-trivial polarization properties [[1], []]. In the nuclear matter, the $S L v$ of an ultrarelativistic neutrino is completely circular polarized.

Due to photon dispersion in most astrophysical environments, the process of radiation has a threshold. For an environment peculiar to neutron stars (NS) the (anti)neutrino threshold energy is [5]

$$
E_{t h} \simeq 28.5 \times \frac{Y_{e}^{2 / 3}}{1-Y_{e}}\left(\frac{10^{38} \mathrm{~cm}^{-3}}{n_{n}}\right)^{1 / 3} \mathrm{TeV},
$$

where $Y_{e}=n_{e} / n_{n} \simeq n_{e} / n_{b}$ is the number of electrons per baryon (the typical values are $Y_{e} \lesssim 0.9$ ). Note that at this high energy range the $S L v$ process is restricted by the W-boson production process.

Summing up, we can infer the following factors for best $S L v$ efficiency: 1) high neutrino energy and high background neutral matter density, 2) low density of the charged matter component, 3) low temperature of the charged matter component (in order to lower the plasmon mass $m_{\gamma}$ and, consequently, the process threshold given by (D)) and 4) considerable extension of the medium.

Among the variety of astrophysical sites, the most appropriate to suit the above conditions is represented by the environment around sGRBs, where the ultra-high energy neutrinos are generated in the relativistic jets and then propagate through the diffuse neutrino wind blown during neutron stars merger. For the $S L v$ radiation time $\tau_{\mathrm{SL} v}$ in the case of a high energy neutrino $\left(E_{V} \sim 10^{12}-10^{18} \mathrm{eV}\right)$ propagating through a GRB media characterized by $\rho=5 \times 10^{3} \mathrm{~g} / \mathrm{cm}^{3}$, $T=0.1 \mathrm{MeV}, Y_{e}=0.01, n_{v} \sim 10^{32} \mathrm{~cm}^{-3}$ [焑] from (四) with $\mu \simeq 2.9 \times 10^{-11} \mu_{B}$ we have:

$$
\tau_{\mathrm{SL} v} \simeq 6.4 \times\left(10^{11}-10^{17}\right) s=2 \times\left(10^{4}-10^{10}\right) \text { years. }
$$

The lower boundary of this range indicates that the total effect of $S L v$ radiation from sGRBs can be substantial if the sufficient number of ultra high-energy neutrinos are born during the event.

This work was supported by the Russian Foundation for Basic Research under grants No. 1602-01023 A and No. 17-52-53133 GFEN_a.

\section{References}

[1] A. Lobanov and A. Studenikin, Phys. Lett. B 564 (2003) 27.

[2] A. Studenikin and A. Ternov, Phys. Lett. B 608 (2005) 107; [hep-ph/0410297].

[3] A. Grigoriev, A. Studenikin and A. Ternov, Phys. Lett. B 622 (2005) 199.

[4] A. Grigoriev, A. Lokhov, A. Studenikin and A. Ternov, Phys. Lett. B 718 (2012) 512.

[5] A. Grigoriev, A. Lokhov, A. Studenikin and A. Ternov, JCAP 11 (2017) 024.

[6] A. Perego at al, Mon. Not. R. Astron. Soc. 4434 (2014) 3134. 